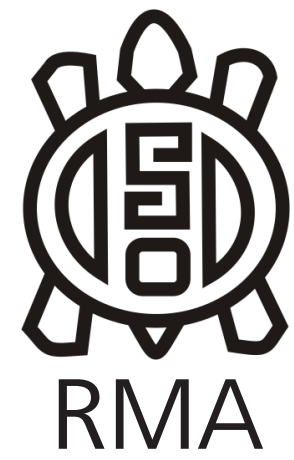

Dossier

\title{
Plantas que inspiran: ensayo de aplicación de categorías posthumanistas a la arqueobotánica del este de Norpatagonia (Argentina)
}

\author{
Plants that inspire: essay of applying of post-humanist categories to \\ archaeobotany in east Norpatagonia (Argentina) \\ Daniela Saghessi*
}

*CONICET, División de Arqueología, Facultad de Ciencias Naturales y Museo, Universidad Nacional de La Plata, Argentina. E-mail: dsaghessi@fcnym.unlp.edu.ar

\begin{abstract}
Resumen
La abundancia de referencias etnohistóricas sobre el uso de plantas por parte de los grupos cazadores-recolectores del nordeste patagónico durante los siglos XVIII y XIX contrasta con el escaso abordaje arqueológico que recibió el tema en las investigaciones del siglo pasado. En los últimos años, este panorama ha comenzado a cambiar con el inicio de investigaciones regionales sistemáticas que incorporan nuevas vías analíticas, como el estudio de microrrestos vegetales, a la arqueología de esta región. En paralelo, desde fines del siglo XX, se está produciendo una apertura teórica en la disciplina, cuyos exponentes actuales más representativos son las corrientes conocidas como "Nuevo materialismo" y "Posthumanismo". El presente ensayo tiene como objetivo proponer casos de aplicación para algunas categorías de corrientes teóricas contemporáneas en arqueología (e.g. teoría ontológica, teoría de los ensambles y teoría de las affordances), a partir de la información disponible sobre el uso de las plantas por los grupos cazadores-recolectores que habitaron el este de Norpatagonia, y en particular el valle del río Negro, durante el Holoceno tardío.
\end{abstract}

Palabras claves: Teoría arqueológica; Cazadores-recolectores; Paleoetnobotánica; Uso de plantas; Río Negro

\begin{abstract}
The abundance of ethnohistorical references on the use of plants by hunter-gatherer groups in the northeast Patagonia along the $18^{\text {th }}$ and $19^{\text {th }}$ centuries contrasts with the scant archaeological approach that the topic received in the investigations of the last century. In recent years, this situation has begun to change with the beginning of systematic regional investigations that incorporate new analytical pathways, such as the study of plant microremains, to the archeology of this region. At the same time, since the end of $20^{\text {th }}$ century, a theoretical opening has been taking place in the discipline, whose current most representative exponents are the currents known as "New materialism" and "Post-humanism". The present essay has as aim to propose application for cases some categories of contemporary theoretical currents in archeology (e.g. ontological theory, assembles theory and affordances theory), based on available information on the use of plants by hunter-gatherer groups that inhabited the northeast Patagonia, and particular Rio Negro valley, along the late Holocene.
\end{abstract}

Key words: Archaeological theory; Hunters-gatherers; Paleoethnobotany; Use of plants; Río Negro

\section{Introducción}

La información arqueológica sobre los grupos cazadoresrecolectores del este de Norpatagonia ha aumentado significativamente durante los últimos años debido, principalmente, a los estudios regionales sistemáticos (e.g. Prates, 2008; Mange, 2019) y a la incorporación de nuevos problemas y metodologías (e.g. isotopos estables en Serna, 2018; y microrrestos vegetales en
Saghessi y López, 2019). Este ensayo ${ }^{1}$ se centrará en el aspecto teórico del quehacer arqueológico. El objetivo es proponer casos de aplicación para algunas categorías de corrientes teóricas contemporáneas en arqueología (e.g. teoría ontológica, teoría de los ensambles, teoría

\footnotetext{
1 El presente ensayo surge a partir del trabajo final del curso de posgrado "Tras la interpretación: nuevos materialismos, ensambles y ontologías en arqueología" dictado por el Dr. Andrés Laguens y desarrollado en la Facultad de Ciencias Naturales y Museo de la Universidad Nacional de La Plata.
} 
de las affordances y arqueología simétrica), a partir de la información disponible y preguntas vigentes sobre el uso de las plantas por los grupos cazadores-recolectores que habitaron el este de Norpatagonia, y en particular el valle del río Negro, durante el Holoceno tardío (ca. 3500/3000 - 300 años AP). De esta manera, se busca reflexionar acerca de cómo las diferentes investigaciones arqueológicas de la región han abordado el uso de plantas y sobre como la incorporación de algunos de los aportes teóricos-conceptuales de estas líneas de pensamiento (e.g. affordances, ontologías, ensambles) podrían contribuir a la interpretación arqueológica del problema.

Las primeras referencias sobre las plantas y sus usos por parte de los grupos cazadores-recolectores patagónicos se encuentran en los diversos informes de viajes elaborados por cronistas, funcionarios, viajeros y misioneros que recorrieron la región desde tiempos de la conquista y la colonización europea en los siglos XVI y XVII (e.g. Fernández de Oviedo y Valdés [1526] citado en Ciampagna y Capparelli, 2012), y sobre todo durante los siglos XVIII y XIX (e.g. Guinnard, [1856-59] 1947; Musters, [1869-70] 1997)(Casamiquela, 1999; Prates, 2009). En dichos trabajos, se realizan descripciones etnográficas de los grupos humanos y se detallan, entre otras cosas, que plantas eran utilizadas, para qué servían y cómo se consumían. Un claro ejemplo es el de los usos y formas de preparación del fruto del algarrobo registrado por numerosos autores, entre ellos Claraz en su viaje al Chubut: "...las comen ya sea crudas o cocidas, ya un poco tostadas en ceniza caliente; después de masticarlas, escupen las semillas y vainas. También hicieron harina. La sustancia alveolar que rodea las semillas contiene azúcar y es harinosa. Se tuestan en un instante, luego se machacan entre piedras, y la sustancia alveolar es la que proporciona la harina. Pero como las vainas, los pedacitos y las semillas están mezclados, se pasa todo por un tamiz. Se come la harina que tiene un sabor dulce, parecido al assamar. Las vainas (...) y las semillas se hierven en agua y lo ingieren (...); pero tiene un gusto demasiado dulce..." (Claraz [1865-66] 1988 citado en Prates, 2009).

En las últimas décadas, el estudio de los restos vegetales arqueológicos ha sido foco de debates relacionados con su correcta denominación. Así, el término "arqueobotánica" quedó reservado para investigaciones que responden a problemas puramente arqueológicos y el término "paleoetnobotánica" para aquellas enfocadas en la relación entre comunidades humanas y vegetales, es decir una etnobotánica del pasado (Archila et al., 2008). En Patagonia los análisis arqueobotánicos se han diversificado en cuanto a los tipos de restos estudiados (e.g. carbón, semillas, frutos, fibras, fitolitos, almidones, polen) y a las líneas de investigación desarrolladas (e.g. estudios de macro y microrrestos, arqueología experimental, nutricionales, paleoetnobotánica, etnobotánica histórica). En el sur de la región, además de varios estudios antracológicos (carbones) (e.g. Piqué i Huerta, 1999; Ancibor y Pérez de Micou, 2002;

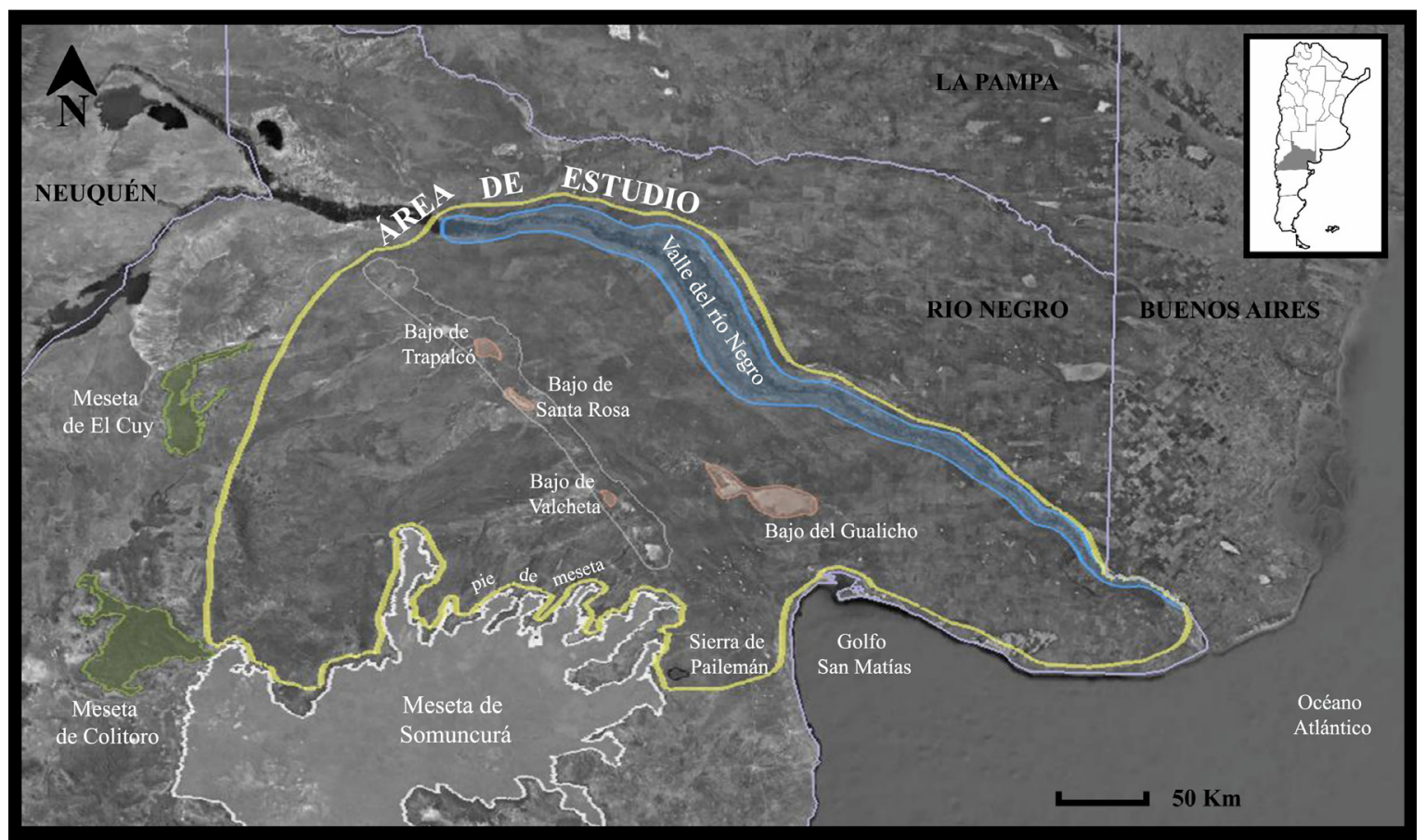

Figura 1. Delimitación del área de estudio donde se muestran los tres sectores: valle del río Negro, pie de la meseta de Somuncurá y, bajos y planicies intermedios.

Figure 1. Delimitation of the study area where showing the three sectors: Río Negro valley, piedmont of the Somuncurá mesa and the intermediate plains and closed basins. 
Marconetto, 2002; Ortega y Marconetto, 2009; Caruso, 2013; Pasqualini, 2015; Ciampagna et al., 2016; Cueto y Andreoni, 2016), son numerosos los trabajos sobre el registro vegetal a partir de fuentes documentales (Nacuzzi y Pérez de Micou, 1983-1985; Caruso et al., 2008; Ochoa y Ladio, 2011) y estudios sobre prácticas de recolección y gestión de plantas entre los grupos cazadores-recolectores (e.g. Berihuete, 2006, 2009; Ciampagna, 2015). Para Norpatagonia andina, se suman además estudios carpológicos (frutos y semillas) (e.g. Llano, 2010; Llano y Andreoni, 2012; Ciampagna y Mange, 2017), de microrrestos (fitolitos y granos de almidón) (e.g. Pérez y Erra, 2011; Lema et al., 2012) y de rendimiento económico y aporte nutricional (e.g. Llano y Ugan, 2010; Llano et al., 2012), los cuales han permitido evaluar el uso alimenticio de diversas plantas silvestres y registrar la presencia de especies domesticadas (maíz -Zea mays-). En el este de Norpatagonia, si bien la información etnohistórica sugiere un uso frecuente y variado de los recursos vegetales (Casamiquela, 1999; Prates, 2009), la información arqueobotánica aún es escasa y se concentra en el sector del valle del río Negro (Capparelli y Prates, 2010 y 2015; Saghessi y López, 2019). Por este motivo, el resto del trabajo se acotará a este último sector.

\section{Área de estudio}

El área de estudio se sitúa en el este de Norpatagonia, e incluye el centro y este de la provincia de Río Negro. Está limitada al norte por la cuenca del río Negro, al sur por el pie de la meseta de Somuncurá, al oeste por las mesetas de El Cuy y de Colitoro (aproximadamente meridiano $69^{\circ}$ O) y al este por la faja costera. Debido a las marcadas diferencias ambientales existentes, se pudieron definir tres sectores: 1) el valle de río Negro, 2) las estribaciones de la meseta de Somuncurá y 3) los bajos y planicies intermedios (e.g. Trapalcó, Santa Rosa, Valcheta, Gualicho) (Figura 1). Los dos primeros asociados con sistemas fluviales permanentes, como son la cuenca del río Negro y el arroyo Valcheta en las estribaciones de Somuncurá; y el tercero con extensas áreas con fuentes efímeras de agua dulce. En términos fitogeográficos el área se ubica principalmente en la provincia del Monte, en el dominio Chaqueño (Cabrera, 1971), y se caracteriza por un clima árido-semiárido (Bruniard, 1982; Mancini et al., 2005). En los tres sectores definidos se reconocen diferencias en cuanto a la cobertura vegetal y disponibilidad de recursos. El valle del río Negro pertenece al distrito del Monte Austral (Cabrera, 1976; León et al., 1998). Su vegetación característica es la estepa arbustiva -o matorral- (Cabrera, 1976; León et al., 1998; Bran et al., 2000); pero además en las zonas bajas donde los suelos son húmedos, contiene formaciones de bosque marginal (Morello, 1958), matorrales ribereños (Frangi y Malacalza, 1978), gran cobertura graminosa y mayor desarrollo de especies con frutos comestibles, sobre todo Geoffroea decorticans y variedades arbóreas de Prosopis
(Balmaceda, 1979).

\section{Las plantas en las investigaciones arqueológicas del este de Norpatagonia}

Los primeros registros sobre sociedades cazadorasrecolectoras de la región y los recursos utilizados corresponden a las fuentes etnohistóricas (ver síntesis en Casamiquela, 1999; Prates, 2009; Salemme y Frontini, 2011; Ciampagna y Capparelli, 2012; entre otros). Además en estos documentos figuran, en mayor o menor medida, observaciones de materiales y sitios arqueológicos. En cuanto a las plantas, la diversidad de especies y usos reconocidos es abundante. Existen referencias sobre el aprovechamiento de todos los órganos vegetales y/o sus partes, es decir hojas, frutos, semillas, tallos, corteza, fibras, raíces, tubérculos, bulbos, rizomas, etc.; tanto sobre usos alimenticios como no alimenticios (combustibles, medicinales, tintóreo, confección de artefactos-e.g. cestería-), y sobre modos de utilización y prácticas asociadas. En términos generales, un gran número de citas refieren al consumo de plantas con órganos subterráneos de almacenamiento (raíces, tubérculos, rizomas y bulbos), siendo algunos de los taxones identificados: Arjona sp., Oxalis sp., Bolax gummifera (Lam.) Spreng., entre otros (Prates, 2009; Ciampagna y Capparelli, 2012).

Las plantas con frutos comestibles son otro grupo muy mencionado, entre las cuales se señalan especies como: (a) Prosopis sp., del cual existen diversas referencias sobre el consumo de sus frutos frescos y la fabricación de bebidas, harinas y productos elaborados y; (b) Condalia microphylla (piquillín) y Geoffroea decorticans (chañar), cuyos frutos constituyeron una fuente habitual de alimentos (e.g. Claraz, [1865-66] 1988; Guinnard, [185659] 1947). Otros géneros de importancia son: Schinus sp. (molle), con una resina apreciada para uso masticatorio y limpieza de la dentadura y como sustancia adhesiva para enmangue de artefactos líticos; y Apium sp. (apio silvestre), citado por sus usos medicinales y alimenticios (e.g. Burmeister, 1888; Claraz, [1865-66] 1988; Musters, [1869-70] 1997). Toda esta información representa una fuente clave para el estudio de los usos de las plantas por parte de los grupos cazadores-recolectores patagónicos, aun cuando las descripciones y nomenclaturas botánicas puedan tener ciertas imprecisiones y los objetivos de los escritos hayan sido diversos.

Desde mediados del siglo XIX comienzan a aumentar la cantidad de observaciones arqueológicas en el área (e.g. Strobel, 1867; Moreno, 1874). A principios del siglo XX, y hasta 1940, los estudios se centraron en la descripción de restos arqueológicos puntuales como por ejemplo las hachas de piedra, los discos grabados y numerosos entierros humanos, del sector del litoral atlántico y del valle inferior del río Negro (e.g. Ambrosetti, 1902; Lehmman-Nitsche, 1909; Outes, 1916; Márquez Miranda, 
1939). En los siguientes treinta años, entre 1940 y 1970, las investigaciones arqueológicas en el área tomaron fuertes influencias de la escuela Histórico-cultural austroalemana. Durante este tiempo, se propone el primer modelo explicativo arqueológico general de la región (e.g. Bórmida, 1964), y la mayoría de los estudios se centraron en la identificación de industrias líticas (e.g. Sanguinetti de Bórmida, 1974). En simultáneo fueron publicados algunos trabajos etnográficos desde concepciones histórico-culturales (Escalada, 1949; Casamiquela, 1965; Nacuzzi, 1998).

A partir de 1980-1990, comienzan a incorporarse en la región nuevos lineamientos teóricos y metodológicos a las investigaciones arqueológicas (e.g. Borrero, 1981; Gradin, 1984; Peronja et al., 1987). Desde mediados de 1990, y sobre todo a principios de los 2000, se inician los primeros proyectos de investigación regionales con nuevas perspectivas teóricas vinculadas tanto a la arqueología procesual como a una postura postprocesual (Shanks y Hodder, 1995). Comienza a hacerse hincapié en estrategias de adaptación, en cambios de subsistencia, y la dinámica poblacional de distintos sectores del este de Norpatagonia (e.g. Borella y Favier Dubois, 2006; Martínez, 2008). A partir de las investigaciones de los últimos quince años en el valle del río Negro, se consolidó la idea de que los grupos cazadores-recolectores que habitaron este sector durante el Holoceno tardío tuvieron una subsistencia basada en la caza y la recolección; y principalmente, en la explotación de diversos recursos faunísticos (ver síntesis en Prates, 2008; Mange, 2019).

En este punto cabe preguntarse acerca de qué sucedió con aquella gran cantidad de especies vegetales descriptas en las fuentes etnohistóricas. Lo cierto es que la abundancia de referencias sobre el empleo de vegetales por parte de los grupos patagónicos permite señalar que las plantas debieron tener más importancia de lo que indican las evidencias arqueológicas. La escasa preservación de evidencia vegetal macroscópica, sumada a la falta de aplicación de técnicas adecuadas para su recuperación, ha conducido a que las menciones sobre restos vegetales recuperados en sitios arqueológicos de la región disminuyeran drásticamente en las investigaciones del siglo pasado. En los últimos años, este panorama ha comenzado a cambiar. Para el sector del valle del río Negro, pueden citarse los estudios de Prates (2008) y Mange (2019) donde se describen brevemente los restos botánicos recuperados, y se los incorporan al análisis arqueológico general. Entre los trabajos centrados en el análisis de restos vegetales se encuentran los de Capparelli y Prates (2010 y 2015) sobre los macrorrestos del sitio arqueológico Angostura 1. Los datos obtenidos permitieron identificar frutos de algarrobo (Prosopis sp., Fabaceae), cuyas vainas se carbonizaron posiblemente de manera accidental mientras eran tostadas en cenizas calientes, como etapa previa a su molienda. También se estableció la presencia de un fruto de mistol (Ziziphus mistol Griseb., Rhamnaceae), que si bien no pudo confirmarse que haya sufrido algún tipo de procesamiento, su hallazgo fuera de su ámbito fitogeográfico nativo permitió inferir posibles contactos con sociedades de áreas septentrionales más alejadas y/o circuitos de movilidad más extensos. Además, actualmente se están realizando estudios de microrrestos vegetales en tártaro dental humano de individuos inhumados en sitios arqueológicos del valle medio del río Negro (Saghessi y López, 2019).

\section{Aplicando categorías posthumanistas}

Uno de los interrogantes aún vigente en cuanto al uso de las plantas refiere a la definición de su rol en la dieta de los grupos cazadores-recolectores que habitaron el área de estudio durante el Holoceno tardío. Como se ha mencionado, existe abundante información documental del tema, sin embargo el abordaje arqueológico de este interrogante no se ha realizado aun de forma sistemática. Una de las posibles causas es la ya aludida escasez de restos botánicos macroscópicos en los sitios arqueológicos de la región, los cuales representan el tipo de evidencia por excelencia para los estudios arqueobotánicos. A comienzos del siglo $X X I$, se produce un incremento de los estudios de microrrestos vegetales, lo que favoreció el acceso a nuevos interrogantes y perspectivas en las investigaciones arqueológicas en otras regiones de Argentina, como el noroeste (NOA) (e.g. Babot, 2004; Korstanje, 2005; Lema, 2009). En Patagonia, y en áreas cercanas como el sur de La Pampa, la incorporación de análisis de fitolitos y granos de almidones a los proyectos de investigación es aún más reciente (e.g. Pérez y Erra, 2011; Lema et al., 2012; Ciampagna, 2014; Musaubach, 2014; Prates et al., 2019).

A raíz de este panorama surge una nueva pregunta más general, que incluye a la anterior, y es cómo abordar las relaciones entre los grupos humanos y las plantas que tuvieron lugar a lo largo de los últimos tres mil años en el centro-este de la provincia de Río Negro; y en este sentido, qué papel tuvieron las plantas en esas relaciones. Una primera opción sería considerar a las plantas como formas acabadas, similares a objetos, las cuales interactúan con los seres humanos sólo para sufrir cambios por uso o consumo (Ford, 1979 y 1985 citado en Lema, 2017; Oslen, 2010). El problema aquí es que las plantas no son entidades cerradas, sino entidades que crecen y se transforman en virtud de las diferentes relaciones con su entorno y con otras entidades. En esta visión, las plantas y los seres humanos son similares. Esto se vincula con lo planteado por Ingold (2008, 2012), donde los artefactos, los humanos y no humanos (plantas y animales) deben ser considerados como "cosas" en lugar de "objetos". La razón es que, desde esta nueva concepción, las "cosas" refieren a formas potenciales, nunca finales, que siempre están en movimiento y 
son parte de los procesos de la vida; lo que implicaría pensar que las "cosas" tienen un rol activo dentro de esas relaciones. En este sentido, es interesante observar como desde otras disciplinas, como la etnobotánica y la paleoetnobotánica, se puede plantear una situación similar en relación al cambio de perspectiva en la concepción del sujeto-objeto de estudio con esta visión, que en arqueología se la situó dentro de la corriente teórica contemporánea de "Nuevos Materialismos". El interés de estas dos disciplinas radica en el estudio de las relaciones plantas-grupos humanos tanto en el pasado como en el presente, las cuales tienen lugar en contextos dinámicos donde ambas partes se influyen mutuamente y se condicionan (Portères, 1966; Alcorn, 1995; Albuquerque, 2016; Lema, 2017).

Otro aporte del "Nuevo Materialismo" es el concepto de "affordances" (Gibson, 1971 a y b citado en Laguens, 2007), traducido al español como "oportunidades" u "ofertas". Este término refiere a distintas posibilidades, u oportunidades, que ofrece una "cosa", y que dependerán de los distintos contextos de relación; es decir que se hace hincapié en una relación situada -depende de quién entre en relación- y en un sentido ecológico. Con respecto a las plantas, podría pensarse como éstas tienen diferentes "affordances", las que influirán su relación con el entorno, con los grupos humanos y otros animales. Un ejemplo específico podría ser la capacidad que tienen algunas plantas de acumular sílice en sus tejidos. Es conocido que algunas especies, como las pertenecientes a la familia de las gramíneas, son más propensas a concentrar sílice que otras debido a ciertas particularidades metabólicas. El ácido monosilícico $\left(\mathrm{H}_{4} \mathrm{SiO}_{4}\right)$ es absorbido en la raíz y viaja por vía xilemática hasta la epidermis de las hojas y los tallos, principalmente, donde se deposita ocupando tanto espacios intra como inter celulares (Piperno, 2006). De este proceso, deriva la formación de silicofitolitos (Zucol, 1992; Pearsall, 2015), uno de los principales microrrestos estudiados en los análisis arqueobotánicos actuales. Así, la posibilidad de que una planta acumule sílice y forme silicofitolitos puede ser considerada como una "affordance" ya que, si bien es una propiedad específica de la planta, esta dependerá, entre otros factores, de la interacción con la cantidad de sílice que haya en el sustrato, la concentración de otros elementos inhibidores de la acumulación de sílice (por ejemplo potasio) en el sustrato, y la existencia de grupos humanos y otros animales que consuman la planta. De manera similar, se ha empleado el término "agencia" para señalar esta postura relacional entre entidades humanas y no humanas; y como un agente, que no necesariamente tiene que ser siempre una persona, puede producir un efecto sobre otro (Gell, 1998; Dobres y Robb, 2000; Olsen, 2003; Latour, 2005).

En resumen un punto central de estas corrientes de pensamiento arqueológico es la idea de romper con la pasividad de todo aquello que no sea humano e incorporar, a través de conceptos como el de "affordances", un contexto relacional situado al análisis arqueológico. Pero que sucede cuando se quiere indagar sobre cómo los grupos de cazadores-recolectores veían, vivían y construían su mundo; y también sobre cómo, en ese mundo, se relacionaban con las plantas, y éstas eran valoradas diferencialmente. Se ha planteado la necesidad de orientar ontológicamente las investigaciones arqueológicas (e.g. Alberti y Marshall, 2009; Alberti, 2016; Lema, 2017). Para lograr esto se ha vuelvo central la teoría del perspectivismo y los estudios sobre, y basados en, el animismo amazónico (e.g. Viveiro de Castro, 2004 y 2010; Rival, 2012; Descola, 2014). Así los relatos etnográficos sobre los mundos de otras personas se convirtieron en recursos básicos para pensar en la alteridad ontológica en arqueología (e.g. Alberti y Marshall, 2009; Lema, 2017).

En el caso de las plantas, los estudios etnobotánicos y etnobotánicos históricos permiten ampliar y sustentar posibles interpretaciones arqueológicas sobre el uso de las plantas en el pasado (e.g. Daly et al., 2016; Lema, 2017; Musaubach et al., 2018). En Norpatagonia, y en áreas cercanas (sur de La Pampa), se han hecho este tipo de estudios con comunidades mapuches y rankulches, cuyos resultados han permitido, entre otras cosas, establecer la continuidad en el uso de algunas plantas desde los hechos registrados por las fuentes etnohistóricas (e.g. Prosopis sp., Prosopis caldenia) y conocer aspectos relevantes sobre la apropiación y el simbolismo que conlleva la utilización de algunas especies vegetales a través de relatos tradicionales (e.g. Ciampagna y Capparelli, 2012; Molares y Ladio, 2015; Ochoa y Ladio, 2015a; Musaubach et al., 2018). Este es el caso de la araucaria, o pehuén (Araucaria araucana), la cual es, y fue, representada como el árbol de la vida, como un símbolo de protección y reparo en los epew (relatos) mapuches (Montes, 2000 citado en Musaubach et al., 2018; Reis et al., 2014; Cortés et al., 2019). Además en diversos documentos etnohistóricos se señala como los piñones de araucaria eran un alimento básico y se consumían frescos y cocidos (e.g. Musters, [1869-70] 1997). Esto lleva a pensar como el análisis arqueológico del uso de las plantas es una parte de un estudio más abarcativo, el del conocimiento botánico tradicional (CBT) de los grupos de cazadoresrecolectores que habitaron el centro-este de la provincia de Río Negro durante el Holoceno tardío. Entendiendo al CBT como un conjunto de conocimientos, prácticas y creencias compartido por una comunidad acerca de las relaciones entre los seres humanos y las plantas (Cotton, 1998; Pochettino et al., 2012), se puede plantear como su estudio permitiría acceder a otras ontologías, a otras formas de conocer y ser o "llegar a ser" con plantas (Daly et al., 2016).

Otro postulado común de estas corrientes teóricas es el de descentrar a las personas dentro de las interpretaciones arqueológicas. Se propone una reorientación ontológica 
que ponga a humanos y no humanos en el mismo nivel. Se ha denominado a este movimiento como "arqueología simétrica", y entre sus representantes se encuentran Bjørnar Olsen, Timothy Webmoor, Christopher Witmore y Michael Shanks. Algunos de sus principios son: 1) las cosas son ensambles, 2) las cosas son participantes, y 3) las cosas son cosas (Wittmore, 2014). Respecto al primer punto, la incorporación del concepto de ensamble, entendido como un conjunto heterogéneo y multitemporal, al análisis arqueológico ha proporcionado una forma diferente de pensar el mundo; de tener una mirada centrada en formas fijas y finales se pasa a contemplar una multiplicidad de elementos que se unen y sostienen todas las cosas diferentes del mundo (Deleuze y Guaitari, 2004). Así se plantea que un ensamble tiene siempre dos ejes principales, el de la composición y el del dinamismo (Lucas, 2012; Harris, 2017). En cuanto al eje de la composición, un ensamble contienen elementos materiales (físicos) y expresivos (signos, gestos, símbolos, entre otros). Mientras que el eje del dinamismo, reconoce como las composiciones de un ensamble siempre se unen (territorialización) y se separan (desterritorialización), ya que los ensambles nunca son fijos. Al mismo tiempo, todos los ensambles están en sí mismos formados por otros ensambles, estos permite tratarlos a diferentes escalas (Fowler, 2017; Harris, 2018).

En relación al tema de este trabajo, podría pensarse a la dieta como un ensamble que está compuesta por diversas cosas: plantas, animales, artefactos (cerámicas, morteros, manos de moler, puntas de flecha) y personas. Estos diferentes conjuntos tendrán cada uno algún proceso histórico específico que los individualice y, a su vez, otros procesos que los vincule con otros ensambles (Lucas, 2012). Un caso concreto podría ser el análisis de tártaro dental humano de individuos inhumados en distintos sitios arqueológicos del valle del río Negro fechados en el Holoceno tardío. Entre los resultados más destacables se encuentran restos de plantas silvestres locales (Prosopis sp., y gramíneas) y domesticadas no locales (Zea mays maíz) (Saghessi y López, 2019). Así, se podría proponer la forma en que las diferentes plantas conformarían al ensamble "dieta". El caso del Prosopis sp. y las gramíneas se consideraría como elementos que territorializan, o estabilizan al ensamble debido a su recurrencia (Harris, 2107, 2018), la cual está registrada en las fuentes etnohistóricas. Por el contrario, el maíz sería visto como un elemento que desterritorializa y que introduce un componente nuevo al ensamble. A la vez que se establece una conexión con otro $u$ otros ensamble/s donde el maíz forma parte, la cual puede ser analizada en una escala diferente a la del hallazgo de los microrrestos. Esto se vincula estrechamente con el interrogante de la dispersión de los cultígenos entre sociedades sin registro de prácticas agrícolas. La hipótesis más sólida, hasta el momento, plantea que el maíz habría ingresado al área como producto de intercambio. La discusión del consumo de especies vegetales en relación al desplazamiento geográfico de los grupos cazadores-recolectores a partir de la información isotópica disponible (Serna, 2018) permitirá aportar información para la resolución de este interrogante. Otros componentes que se conectan en este ensamble "dieta" son los diversos artefactos que se involucran en los diferentes actos de preparación y cocción de las comidas, los cuales podrían en sí mismo ser vistos como otro ensamble cuyos componentes tienen ciertas características expresivas que codifican la identidad del conjunto. Este podría ser el caso de la cerámica decorada del sitio Loma de los Muertos (Di Prado, 2015).

Además se puede visualizar como el ensamble "dieta" agrupa a otros ensambles, algunos más permanentes o duraderos y otros, al contrario, más efímeros. Esto dependerá de los diferentes procesos de contención y de individualización, y la consecuente creación de centros de gravedad para ese ensamble (Yarrow, 2003; Lucas, 2012; Wittmore, 2014; Harris, 2018). En el ejemplo anterior podría ser algún sector del valle del río Negro donde se encontraron y vincularon recurrentemente grupos humanos, plantas (Prosopis, gramíneas y otras) y animales, y a través de estas conexiones se estableció un patrón que permitió que ese ensamble "dieta" dure más tiempo. Otro ejemplo podría ser el consumo de plantas con órganos subterráneos de almacenamiento, del cual existen registros desde las fuentes etnohistóricas, hasta estudios etnobotánicos recientes con comunidades mapuches (Ochoa y Ladio, 2011, 2015b). Por lo que podría considerarse a estas plantas como componentes duraderos del ensamble "dieta". Por último, un análisis de la variabilidad temporal de estos ensambles, a través de diferentes vías analíticas -e.g. arqueológica y etnohistórica-, dentro de una escala espacial mayor, como la del área de estudio, podría ayudar a descifrar las distintas razones de las prácticas del ensamble y sus límites (Swenson, 2018).

\section{A modo de cierre}

En este ensayo, se propusieron casos concretos de aplicación para algunas categorías de corrientes teóricas en arqueología, como la teoría de las affordances, la teoría ontológica y la teoría de los ensambles. Respecto al objetivo propuesto vinculado al uso de las plantas por los grupos cazadores-recolectores que habitaron el este de Norpatagonia durante el Holoceno tardío surgen las siguientes reflexiones:

- El problema del uso de las plantas ha recibido un abordaje dispar a lo largo de la historia de las investigaciones arqueológicas de la región. Las abundantes descripciones acerca de las especies vegetales de la mano de las fuentes etnohistóricas contrastan con el escaso desarrollo del tema en las investigaciones del siglo pasado. Los estudios actuales están empezando a cambiar este panorama con la incorporación de nuevas vías analíticas, como el estudio 
de microrrestos vegetales.

- Los casos-problemas y los interrogantes tratados en la última sección muestran un terreno propicio para la incorporación de estas líneas de pensamiento arqueológico. Sin embargo, también exponen el carácter preliminar de este ensayo y la necesidad de ampliar los estudios arqueobotánicos a nivel regional. Esto a futuro ayudará no sólo a contrastar las observaciones etnohistóricas sobre la diversidad de plantas utilizadas en el pasado, sino además reevaluar los planteos aquí propuestos en términos espaciales y temporales.

- Por último, considero que la utilización de un enfoque teórico-metodológico paleoetnobotánico en los próximos análisis sobre el uso de las plantas en el área de estudio permitirá, como se evidencia en otros sectores de Patagonia, no sólo la identificación taxonómica de los restos vegetales recuperados, sino también explorar otras aristas de este fenómeno complejo.

\section{Agradecimientos}

Este trabajo se llevó a cabo en el marco del plan de trabajo de una beca interna Doctoral de CONICET y de los proyectos: "Continuidad y cambio en los patrones de explotación de recursos en el centro de Río Negro durante el Holoceno tardío" (PICT 2015-3645) y "Arqueología, cronología y paleo-ambiente en las mesetas del centroeste de Río Negro" (PIP-CONICET N²44/15) -ambos dirigidos por el Dr. Luciano Prates-; a los cuales doy las gracias. También quiero agradecer al Dr. Andrés Laguens por sus buenos comentarios en el trabajo final del curso de posgrado "Tras la interpretación: nuevos materialismos, ensambles y ontologías en arqueología" y su invitación a participar de este dossier; a la Dra. Laura López y al Dr. Luciano Prates por su revisión crítica del manuscrito; y a los evaluadores anónimos por sus sugerencias y comentarios, los cuales han contribuido a mejorar este ensayo.

\section{Bibliografía}

Alberti, B. (2016). Archaeologies of Ontology. Annual Review of Anthropology, 45, 163-179.

Alberti, B. y Marshall, Y. (2009). Animating archaeology: local theories and conceptually open-ended methodologies. Cambridge Archaeological Journal, 19(3), 344-56

Albuquerque, U.P., y Alves, R.R.N. (Eds.). (2016). Introduction to ethnobiology. Boston, United States: Springer.

Alcorn, J.B. (1995). The scope and aims of ethnobotany in a developing world. In: Evans Schultes, R., and von
Reis, S. (Eds.), Ethnobotany: evolution of a discipline (23-39). Portland, United States: Discorides Press.

Ambrosetti, J.B. (1902). Arqueología argentina: un nuevo Pillan-Toki (hacha votiva de piedra). Revista del Museo de La Plata, 10, 265-269.

Ancibor, E., y Pérez de Micou, C. (2002). Reconocimiento de especies vegetales combustibles en el registro arqueológico de la estepa patagónica. En: Pérez de Micou C. (Comp.) Plantas y Cazadores en Patagonia (15-31). Ciudad autónoma de Buenos Aires, Argentina: Universidad de Buenos Aires, Facultad de Filosofía y Letras.

Archila, S., Giovannetti, M., y Lema, V. (Eds.) (2008). Arqueobotánica y Teoría arqueológica. Discusiones desde Suramérica. Bogotá, Colombia: Ediciones Uniandes.

Babot, M. P. (2004). Tecnología y utilización de artefactos de molienda en el Noroeste Prehispánico (Tesis Doctoral). Universidad Nacional de Tucumán, Tucumán-Argentina.

Balmaceda, A. (1979). Vegetación. En: Estudio de clima, geomorfología, suelos, vegetación y erosión de la zona del área correspondiente a la Hoja Topográfica 39L, Cubanea-San Javier (Río Negro). Comisión de Investigaciones Científicas, Río Negro-Argentina.

Berihuete, M. (2006). Aportaciones de la carpología al análisis de la gestión de recursos vegetales en las sociedades cazadoras recolectoras: el grupo Selknam de Tierra del Fuego (Argentina) (Trabajo de Investigación del Tercer ciclo). Universidad Autónoma de Barcelona, Barcelona-España.

Berihuete, M. (2009). El papel de los recursos vegetales no leñosos en las economías cazadoras recolectoras: propuesta para el estudio de su gestión: el caso del estudio de Tierra del Fuego (Argentina) (Tesis Doctoral). Universidad Autónoma de Barcelona, BarcelonaEspaña.

Borella, F. y Favier Dubois, C. (2006). Proyecto arqueológico en la costa del Golfo San Matías (Pcia. de Río Negro). En: Politis, G. (Ed.), INCUAPA 10 años. Perspectivas contemporáneas en la arqueología pampeana y norpatagónica (9-20). Olavarría, Buenos Aires, Argentina: Serie Monográfica INCUAPA.

Bórmida, M. (1964). Arqueología de la costa norpatagónica. Trabajos de Prehistoria, 16, 7-108.

Borrero, L.A. (1981). La economía prehistórica de los pobladores del Alero de los Sauces (Neuquén, Argentina). Trabajos de Prehistoria, 1, 111-124. 
Bran, D. E., Ayesa, J.A., y Lopez, C. (2000). Áreas ecológicas de Río Negro. Laboratorio de teledetecciónSIG, INTA, Bariloche. Disponible en: http://sipan.inta. gob.ar/productos.htm

Bruniard, E.D. (1982). La diagonal árida argentina: un límite climático real. Revista Geográfica, 95, 5-20.

Burmeister, C. (1888). Últimas exploraciones en Patagonia. Buenos Aires, Argentina: Imprenta de J. A.

Cabrera, A.L. (1971). Fitogeografía de la República Argentina. Boletín de la Sociedad Argentina de Botánica, 14 (1-2), 1-42.

Cabrera, A.L. (1976). Regiones fitogeográficas argentinas. Enciclopedia Argentina de Agricultura y Jardinería. Buenos Aires, Argentina: ACME.

Capparelli, A., y Prates, L. (2010). Identificación especifica de frutos de algarrobo (Prosopis spp., Fabaceae) y Mistol (Ziziphus mistol Griseb, Rhamnaceae) en un sitio arqueológico de Patagonia. Tradiciones $y$ Transformaciones en Etnobotánica, 13-19.

Capparelli, A., y Prates, L. (2015). Explotación de frutos de algarrobo (Prosopis spp.) por grupos cazadores recolectores del Noreste de Patagonia. Chungará. (Arica), 47(4), 549-563.

Casamiquela, R.M. (1965). Rectificaciones y ratificaciones hacia una interpretación definitiva del panorama etnológico de la Patagonia y área septentrional adyacente. Instituto de Humanidades, Universidad Nacional del Sur. Bahía Blanca-Buenos Aires-Argentina.

Casamiquela, R.M. (1999). Proyecto etnobotánica de la Patagonia -informe preliminar-. Aspectos Técnicos, Culturales, Políticos y Legales de la Bioprospección en Argentina, 91-134.

Caruso, L. (2013). Los recursos vegetales en arqueología, estrategias de muestreo y estudio del material leñosos. Buenos Aires, Argentina: Editorial Dunken.

Caruso, L., Mansur, E., y Piqué, R. (2008). Voces en el bosque: uso de recursos vegetales entre cazadores recolectores de la zona central de Tierra del Fuego. Darwiniana, 46(2), 202-212.

Ciampagna, M.L. (2015). Estudio de la interacción entre grupos cazadores recolectores de Patagonia y las plantas silvestres: el caso de la costa norte de Santa Cruz durante el Holoceno medio y tardío (Tesis Doctoral). Facultad de Ciencias Naturales y Museo, Universidad Nacional de La Plata. Buenos Aires, Argentina.
Ciampagna, M. L., Ambrústolo, P., y Zubimendi, M. Á. (2016). Estudios antracológicos en abrigos rocosos de la costa norte de Santa Cruz (Patagonia, Argentina): análisis de los sitios El Oriental y Alero 4. Intersecciones en antropología, 17, 341-352.

Ciampagna, M.L., y Capparelli, A. (2012). Historia del uso de las plantas por parte de las poblaciones que habitaron la Patagonia Continental Argentina. Cazadores Recolectores del Cono Sur, 6, 45-75.

Ciampagna, M. L., y Mange, E. (2017). Primeros estudios arqueobotánicos del sitio Cueva Epullán chica (depto. Collón Curá, provincia del Neuquén). En: Haro, M.T.; Rocchietti, A.M.; Runcio, M.A.; Fernández, M.V. y Hernández de Lara, O. (Eds.) Anti Latinoamérica: una mirada desde el presente hacia el pasado (167-183). Ciudad Autónoma de Buenos Aires, Argentina: Aspha Ediciones.

Claraz, G. [1865-66] (1988). Viaje al río Chubut: Aspectos naturalísticos y etnológicos. Buenos Aires, Argentina: Ediciones Continente.

Correa, M. (1984). Flora Patagónica. Buenos Aires, Argentina: Colección Científica del INTA.

Cortés, J., Ugalde, I., Caviedes, J., y Tomás Ibarra, J.T. (2019). Semillas de montaña: recolección, usos y comercialización del piñón de la araucaria (Araucaria araucana) por comunidades Mapuche-Pewenche del sur de los Andes. Pirineos, 174, 0-48.

Cotton, C.M. (1998). Ethnobotany. Principles and applications. London, England: Wiley \& Sons.

Cueto, M., y Andreoni, D. (2016). Análisis preliminar de la gestión de restos vegetales en ocupaciones del Pleistoceno final /Holoceno temprano en la Meseta Central de Santa Cruz. En: F. Mena (ed.) Arqueología de Patagonia: de mar a mar (430-440). Coyhaique, Chile: CIEP/Ñire Negro Ediciones.

Daly, L., French, K., Miller, T.L., y Eoin, L.N. (2016). Integrating ontology into ethnobotanical research. Journal of Ethnobiology, 36(1), 1-9.

Deleuze, G. y Guattari F. (2004). AThousand Plateaus: Capitalism and schizophrenia. London, England: Continuum.

Descola, P. (2014). Modes of being and forms of predication. Hau: Journal of Ethnographic Theory, 4 (1): 271-280

Di Prado, V.S. (2015). Estudio comparativo de las prácticas de elaboración y uso de la alfarería prehispánica del centro-este de Argentina desde una 
perspectiva macrorregional (Tesis Doctoral). Facultad de Ciencias Naturales y Museo, Universidad Nacional de La Plata, Buenos Aires, Argentina.

Dobres, M.A., y Robb, J.E. (2000). Agency in Archaeology. London, England: Psychology Press.

Escalada, F.A. (1949). El complejo "tehuelche": estudios de etnografía patagónica. Coni, Buenos Aires.

Fowler, C. (2017). Relational Typologies, Assemblage Theory and Early Bronze Age Burials. En: Cambridge Archaeological Journal, 27 (1), 95-109.

Frangi, J. L. y Malacalza, L. (1978). Estudio económico para el desarrollo integral del valle del río Negro: informe ecológico de consultoría para el Ing. Conrado Bauer, ms., (disponible en División Arqueología, FCNyM-UNLP).

Gell, A. (1998). Art and Agency. Oxford, England: Clarendon Press.

Gradin, C. (1984). Investigaciones arqueológicas en Casa de Piedra. Dirección General de Cultura y Ente Ejecutivo Casa de Piedra, Pcia. de La Pampa.

Guinnard A. [1856-59] (1947). Tres años de esclavitud entre los patagones. Relatos de mi cautiverio. Buenos Aires, Argentina: Espasa Calpe.

Harris, O.T.J. (2017). Assemblages and Scale in Archaeology. Cambridge Archaeological Journal, 27:1, 127-129.

Harris, O.T.J. (2018). More than representation: multiscalar assemblages and the Deleuzian challenge to archaeology. History of the Human Sciences, 31 (3), 1-21.

Ingold, T. (2008). When ANT meet SPIDER: Social theory for arthropods. In: Knappett, C., and Malafouris, L. (Eds.), Material Agency. Towards a Non-Anthropocentric Approach. Boston, United Stated: Springer.

Ingold, T. (2012). Toward an Ecology of Materials. Annual Review of Anthropology, 41, 427-442.

Korstanje, M.A. (2005). La organización del trabajo en torno a la producción de alimentos en sociedades agropastoriles formativas (Provincia de Catamarca, República Argentina) (Tesis Doctoral). Universidad Nacional de Tucumán, Tucumán-Argentina.

Laguens, A.G. (2007). Objetos en objetos: hacia un análisis relacional de lo estético en arqueología. Antiquitas, 1(1), 1-9.
Latour, B. (2005). Reensamblar lo social. Una introducción a la teoría del actor-red. Buenos Aires, Argentina: Editorial Manantial.

Lehmann-Nitsche, R. (1909). Hachas y placas para ceremonias procedentes de Patagonia. Revista del Museo de La Plata, 16 (3), 204-240.

Lema, V. (2009). Domesticación vegetal y grados de dependencia ser humano-planta en el desarrollo cultural prehispánico del noroeste argentino (Tesis Doctoral). Facultad de Ciencias Naturales y Museo, Universidad Nacional de La Plata. Buenos Aires, Argentina.

Lema, V. (2017). Al toro ¿por las astas? Reflexiones sobre aproximaciones teóricas y metodológicas a la temática de la domesticación en el Área Andina Meridional. En: Casas Fernández, A., Torres Guevara, J., y Parra Rondinel, F. (Eds.) Domesticación y Agricultura en el Nuevo Mundo: historias y perspectivas del manejo de recursos genéticos (151-176). Universidad Nacional Agraria la Molina, Universidad Nacional Autónoma de México.

Lema, V., Della Negra, C., y Bernal, V. (2012). Explotación de recursos vegetales silvestres y domesticados en Neuquén: implicancias del hallazgo de restos de maíz y algarrobo en artefactos de molienda del Holoceno tardío. Magallania (Punta Arenas), 40 (1), 229-249.

León, R., Bran, D., Collantes, M., Paruelo, J., y Soriano, A. (1998). Grandes unidades de vegetación de la Patagonia extraandina. Ecología Austral, 8, 125-144.

Llano, C. (2010). Aprovechamiento de los Recursos Vegetales entre las Sociedades Cazadoras - Recolectoras del Sur de Mendoza (Tesis Doctoral). Universidad Nacional del Comahue. Río Negro, Argentina.

Llano, C., y Andreoni, D. (2012). Caracterización espacial y temporal en el uso de los recursos vegetales entre los grupos cazadores-recolectores del sur Mendocino durante el Holoceno. Paleoecología humana en el sur de Mendoza: perspectivas arqueológicas, 57-84.

Llano, C., y Ugan, A. (2010). Rendimiento Económico de plantas silvestres del sur de Mendoza: valores nutricionales, costos de manejo e interpretación del registro arqueológico. Tradiciones y transformaciones en Etnobotánica, 44-48.

Llano, C., Ugan, A., Guerci, A., y Otaola, C. (2012). Arqueología experimental y valoración nutricional del fruto de algarrobo (Prosopis flexuosa): inferencias sobre la presencia de macrorrestos en sitios arqueológicos. Intersecciones en antropología, 
13(2), 513-524.

Lucas, G. (2012). Understanding the Archaeological record. Cambridge University Press, 124-168.

Mancini, M. V., Paez, M. M., Prieto, A. R., Stutz, S., Tonello, M. y Vilanova, I. (2005). Mid-Holocene variability reconstruction from pollen records (32-52 S, Argentina). Quaternary international, 132, 47-59.

Mange, E. (2019). Investigaciones arqueológicas en la margen sur del valle medio-superior del río Negro (pcia. de Río Negro) (Tesis Doctoral). Facultad de Ciencias Naturales y Museo, Universidad Nacional de La Plata. Buenos Aires, Argentina.

Marconetto, B. (2002). Análisis de los vestigios de combustión de los sitios Alero Don Santiago y Campo Moncada. En: Pérez de Micou C. (Comp.). Plantas y cazadores en Patagonia (33-51). Universidad de Buenos Aires, Facultad de Filosofía y Letras, Ciudad autónoma de Buenos Aires, Argentina.

Márquez Miranda, F. (1939). Los tokis. A propósito de un nuevo "toki" de la araucanía. Notas del Museo de La Plata, 4 (11), 17-45.

Martínez, G. (2008). Arqueología del curso inferior del río Colorado: estado actual del conocimiento e implicaciones para la dinámica poblacional de cazadores recolectores pampeano-patagónicos. Revista de Arqueología, 3, 71-92.

Molares, S., y Ladio, A. H. (2015). Complejos vegetales comestibles y medicinales en la Patagonia Argentina: sus componentes y posibles procesos asociados. Boletín Latinoamericano y del Caribe de Plantas Medicinales y Aromáticas, 14(3), 237-250.

Morello, J. (1958). La provincia fitogeográfica del Monte. Opera Lilloana, 2, 151-155.

Moreno, F.P. (1874). Description des cimetiéres et paraderos prehistoriques de Patagonie. Revue d'Antropologie, 3, 72-90.

Musaubach, M.G. (2014). Estudios Arqueobotánicos en Sociedades Cazadoras-recolectoras de Ambientes Semiáridos. Análisis de Microrrestos Vegetales en Contextos Arqueológicos de Pampa Occidental (Argentina) (Tesis Doctoral). Facultad de Filosofía y Letras, Universidad Nacional de Buenos Aires. Buenos Aires, Argentina.

Musaubach, M.G., Di Biase, A.R.S., y Beron, M.A. (2018). De epew y foikes: un análisis etnobotánico y etnohistórico sobre las plantas en los pueblos mapuche y rankulche. Quinto Sol, 22(2), 1-24.
Musters, G.C. [1869-1970] (1997). Vida entre los patagones. Buenos Aires, Argentina: Editorial El Elefante Blanco.

Nacuzzi, L.R. (1998). Identidades impuestas. Tehuelches, aucas y pampas en el norte de la Patagonia. Buenos Aires, Argentina: Colección Tesis Doctorales, Sociedad Argentina de Antropología.

Nacuzzi, L., y Pérez de Micou, C. (1983-85). Los recursos vegetales de los cazadores de la cuenca del Río Chubut. Cuadernos del Instituto Nacional de Antropología y Pensamiento Latinoamericano, 5, 407-423.

Ochoa, J.J., y Ladio, A.H. (2011). Pasado y presente del uso de plantas silvestres con órganos de almacenamiento subterráneos comestibles en la Patagonia. Bonplandia, 20(2), 265-285.

Ochoa, J.J., y Ladio, A.H. (2015a). Plantas silvestres con órganos subterráneos comestibles: transmisión cultural sobre recursos subutilizados en la Patagonia (Argentina). Boletín Latinoamericano y del Caribe de Plantas Medicinales y Aromáticas, 14(4), 287 -300 .

Ochoa, J.J., y Ladio, A.H. (2015b). Panorama de los recursos alimentarios subvalorados y olvidados de la Patagonia: las plantas silvestres con órganos de almacenamiento subterráneos. Gaia Scientia, 9(3), 105-119.

Olsen, B. (2003). La cultura material después del texto: re-asociando las cosas. Norwegian Archaeological Review, 36(2), 1-19.

Olsen, B. (2010). In defense of things: archaeology and the ontology of objects. Lanham, United States: Altamira Press.

Ortega, F., y Marconetto, B. (2009). Una discusión "encendida". Primeros resultados de los análisis de vestigios de combustión en concheros de la costa norte de la Provincia de Río Negro: una primera aproximación. En: Salemme M, Santiago F, Álvarez M, Piana E, Vázquez M, Mansur E (Eds.) Arqueología de la Patagonia, Una mirada desde el último confín (1141-1148). Ushuaia, Tierra del Fuego, Argentina: Editorial Utopías.

Outes, F.F. (1916). Las hachas insignias patagónicas. Examen crítico del material conocido y descripción de nuevos ejemplares. Buenos Aires, Argentina: Edición privada.

Pasqualini, S. (2015). Utilización de recursos leñosos y uso del espacio en el noroeste de Santa Cruz a partir del Holoceno tardío: un análisis antracológico de los 
sitios Alero Los Guanacos 1 y Cañadón Guitarra 3. Arqueología, 21(1), 155-156.

Pearsall, D.M. (2015). Paleoethnobotany: a handbook of procedures. Walnut Creek CA, United States: Left Coast Press.

Pérez A., y Erra G. (2011). Identificación de maíz en vasijas recuperadas de la Patagonia Noroccidental Argentina. Magallania (Punta Arenas), 39(2), 309316.

Peronja A., Sánchez y Juliá, E., y López, A. (1987). Prospecciones arqueológicas sobre costa de río, con presencia de bivalvos, Provincia de Río Negro. En: Actas de las Primeras Jornadas de Arqueología de Patagonia (243-248). Trelew, Chubut, Argentina.

Piperno, D., (2006). Phytoliths. A Comprehensive Guide for Archaeologist and Paleoecologist. Lanham, United States: Altamira Press.

Pique I Huerta, R. (1999). Producción y uso del combustible vegetal: una evaluación arqueológica. Universidad Autónoma de Barcelona. Consejo Superior de Investigaciones Científicas, Treballs D'Etnoarqueologia, 3, Madrid.

Pochettino, M.L., Puentes, J.P., Buet Costantino, F., Arenas, P.M., Ulibarri, E.A., y Hurrell, J.A. (2012). Functional Foods and Nutraceuticals in a Market of Bolivian Immigrants in Buenos Aires (Argentina). Evidence-Based Complementary and Alternative Medicine, 1-14.

Portères, R. (1966). Aspects de l'ethnobotanique comme discipline scientifique affirmée. Journal d'agriculture traditionnelle et de botanique appliquée, 13(12), 701704.

Prates, L. (2008). Los indígenas del río Negro. Un enfoque arqueológico. Buenos Aires, Argentina: Colecciones Tesis Doctorales, Sociedad Argentina de Antropología.

Prates, L. (2009). El uso de los recursos por los cazadoresrecolectores posthispánicos de Patagonia continental y su importancia arqueológica. Relaciones de la Sociedad Argentina de Antropología, 34, 201-229.

Prates, L., Serna, A., Mange, E., López, L., Romano, V., Di Lorenzo, M., Saghessi, D., y González, V. (2019). Ocupaciones residenciales y entierros humanos en Negro Muerto 3 (Valle del Río Negro, Norpatagonia). Magallania (Punta Arenas), 47(1), 159-176.

Reis, M. Sedrez dos, A. Ladio, and N. Peroni. (2014).
Landscapes with Araucaria in South America: evidence for a cultural dimension. Ecology and Society, 19(2), 43.

Rival, L. (2012). Animism and the meanings of life: reflections from Amazonia. In: Brightman, M., Grotti, V.E., and Ulturgasheva, O. (Eds.) Animism in rainforest and tundra: personhood, animals, plants and things in contemporary Amazonia and Siberia (69-81). Oxford, England: Berghahn Books.

Saghessi, D. y López, M.L. (2019). Estudio de microrrestos vegetales en tártaro dental humano de sitios arqueológicos del valle del río Negro. En: Libro de Resúmenes de XX Congreso Nacional de Arqueología Argentina (260-261). Universidad Nacional de Córdoba, Córdoba, Argentina.

Salemme, M., y Frontini, R. (2011). The exploitation of Rheidae in Pampa and Patagonia (Argentina) as recorded by chroniclers, naturalists and voyagers. Journal of Anthropological Archaeology, 30 (4), 473-483.

Sanguinetti de Bórmida, A.C. (1974). Investigación arqueológica en la Loma de la Lata, Planicie Banderita y bajo de Mari Menuco (provincia de Neuquén). Relaciones (N.S), 8, 289-311.

Serna, A. (2018). Interacciones humanas en el noreste de Patagonia durante el Holoceno tardio: un enfoque bioarqueológico (Tesis Doctoral). Facultad de Ciencias Naturales y Museo, Universidad Nacional de La Plata. Buenos Aires, Argentina.

Shanks, M. y Hodder, I. (1995). Processual, postprocessual and interpretative archaeologies. In: Hodder, I., Shanks, M., Alexandri, A., Buchli, V., Carman, J., Last, J., and Lucas, G. (Eds.) Interpreting Archaeology: finding meaning in the Past (3-29). London, England: Routledge.

Strobel, P. (1867). Paradero prehistorici in Patagonia. Atti della società Italiana de Scienze Naturali, 10: 167-171.

Swenson, E.R. (2018). Assembling the Moche: the power of temporary gatherings on the North Coast of Peru. World Archaeology, DOI: 10.1080/00438243.2018.1474132

Viveiros de Castro, E. (2004). Exchanging Perspectives. The Transformation of Objects into Subjects in Amerindian Ontologies. Common Knowledge, 10 (3), 463-484.

Viveiros de Castro, E. (2010). Metafísicas Caníbales: Líneas de Antropología Postestructural. Buenos Aires, Argentina: Katz Editores. 
Witmore, C. (2014). Archaeology and the new materialisms. The Journal of Contemporary Archaeology, $1,2,1-44$.

Yarrow, T. (2003). Artefactual Persons: The Relational
Capacities of Persons and Things in the Practice of Excavation. Norwegian Archaeological Review, 36(1), 65-73.

Zucol, A.F. (1992). Microfitolitos: I. Antecedentes y terminología. Ameghiniana, 29(4), 353-362. 